\title{
Simple expressions for performance parameters of complex filters, with applications to super-Gaussian phase filters
}

\author{
Silvia Ledesma \\ Departamento de Física, Facultad de Ciencias Exactas y Naturales, Universidad de Buenos Aires, 1428 Buenos Aires, Argentina \\ Juan Campos, Juan Carlos Escalera, and María J. Yzuel \\ Departamento de Física, Universidad Autónoma de Barcelona, 08193 Bellaterra, Spain
}

Received October 30, 2003

\begin{abstract}
To study the three-dimensional (3-D) behavior produced by complex filters, we have extended the expressions for the axial and the transverse gain to the case in which the best image plane is not near the paraxial focus. Super-Gaussian phase filters are proposed to control the 3-D image response of an optical system. Super-Gaussian phase filters depend on several parameters that modify the shape of the phase filter, producing tunable control of the 3-D response of the optical system. The filters are capable of producing a wide range of optical effects: transverse superresolution with high depth of focus, 3-D superresolution, and transverse apodization with different axial responses. (C) 2004 Optical Society of America

OCIS codes: $110.0110,220.0220,350.5730,220.1230$.
\end{abstract}

The three-dimensional (3-D) response of an optical system must be very different depending on its application. A common goal in many optical systems, for instance, in optical storage, ${ }^{1}$ is to improve transverse resolution. Nevertheless, in most cases, a specific axial behavior is needed. For instance, in photolithography a high depth of focus (DOF) is also required. To achieve transverse superresolution, both in photolithography and in optical storage, very short wavelengths and high numerical apertures (NAs) are used. This has two effects: on one side the optical system must be well corrected (which is difficult), and on the other high NA reduces the $\mathrm{DOF}$ (DOF is related to $\lambda / \mathrm{NA}^{2}$ ). For this reason different superresolution techniques ${ }^{2,3}$ have been proposed as ways to relax the optical system requirements or simply to improve the resolution of an optical system by providing enough DOF. In other systems, such as scanning microscopy, transverse and axial superresolution is desirable to obtain 3-D images. ${ }^{4,5}$

There are different methods that have been used to modify the impulse response of an optical system. Annular transmission pupils have been widely used to improve transverse and (or) axial resolution. $^{4-6}$ Continuously varying amplitude filters have also been investigated to produce transverse superresolution and (or) high DOF. ${ }^{3,6,7}$ However, in recent years, phase-only filters have been studied to check whether they can improve the performance of amplitude-only filters. ${ }^{1}$ In one approach, binary phase filters are used ${ }^{1}$ to obtain elements that are easy to produce. In other works continuous phase filters have been investigated, ${ }^{8,9}$ but in some cases although transverse superresolution is obtained it is produced by very complex phase masks. ${ }^{9}$ In a recent study $^{8}$ a simple continuous phase filter was used to obtain transverse superresolution.

In this Letter we propose super-Gaussian phase filters that have a shape that depends on four parame- ters. These parameters give us enough degrees of freedom to modify the whole $3-\mathrm{D}$ image response in a controlled way. The filters are capable of producing a wide range of optical effects: they can produce transverse superresolution that increases the DOF, 3 -D superresolution, and even transverse apodization with different axial responses. To our knowledge the capability of these phase filters has not been studied in previous works.

The super-Gaussian phase filters that we propose have a transmission $Q(t)=\exp [i \varphi(t)]$, where $\varphi(t)$ is defined as

$$
\varphi(t)=a \exp \left\{-\left[\left(t-t_{0}\right) / \Omega\right]\right\}^{2 \alpha} .
$$

Here $a$ is a parameter that controls the global phase height of the filter, $t$ is equal to the square of the radial coordinate $r, t_{0}$ is the position of the maximum of the phase distribution (in coordinate $t$ ), $\Omega$ is the width, and $\alpha$ is the order of the super-Gaussian ring. By changing the value of $\Omega$ we can get phase profiles that go from narrow rings (for instance, at $\Omega=0.1$ ) to smooth profiles that almost cover the entire pupil (for instance, at $\Omega=1$ ). For $\alpha=1$ the phase filter becomes an annular Gaussian ring. In the limit $\alpha \rightarrow \infty$ the superGaussian ring is identical to an annular phase filter, but in practice for $\alpha=5$ the function is similar to a ring. The super-Gaussian profiles used here are similar to the ones proposed in Ref. 7, but now they are phase profiles, and the variable used is $t=r^{2}$ for better axial predictability. ${ }^{10}$ Note that the performance of super-Gaussian phase filters is not particularly affected by the choice of the $\alpha$ value.

In Ref. 6 a simple theory of focal behavior was proposed. In that work superresolution factors $G_{T}$ and $G_{A}$, for the transverse and axial performance, respectively, were defined for amplitude-only filters. These superresolution factors are a useful tool for filter design. These factors were generalized by de Juana et al. ${ }^{8}$ for phase filters, also working near the paraxial 
plane. The filters studied here can shift the best image plane (BIP) away from the BIP without a filter. In this Letter we generalize the gain parameters for any complex filter in the surroundings of the shifted focus.

The method that we propose has the following steps: first we calculate the axial response produced by the filter, second we search for the maximum of the on-axis intensities, and then we develop up to second order the superresolution factors around that point. Note that the second step of the procedure is valid for an arbitrary shift of focus from the BIP without a filter; meanwhile the last step is valid in the area surrounding the shifted focus.

Let us call $Q(t)$ the transmission function of an arbitrary pupil. Then, the electromagnetic field along the axis will be

$$
U(0, u)=\int_{0}^{1} Q(t) \exp (i u t / 2) \mathrm{d} t
$$

where $u$ is the axial coordinate centered at the focal plane or the BIP without a filter. As we mentioned above, in the case of some phase filters this distribution could reach its maximum value in a position displaced from this plane. We evaluate $\left|U(o, u)^{2}\right| \mathrm{nu}-$ merically from Eq. (2) along the axis, and we find the position $u_{\max }$ where the axial intensity is maximum. Once we have found $u_{\max }$ we calculate the superresolving gains by use of the expansions of the axial and transverse intensities around this point.

For the axial response we consider a second-order expansion of Eq. (2) around $u_{\max }$ :

$$
\begin{aligned}
U(0, u) \cong & \int_{0}^{1} Q(t) \exp \left(i u_{\max } t / 2\right)\left[1+(i t / 2)\left(u-u_{\max }\right)\right. \\
& \left.-\left(t^{2} / 8\right)\left(u-u_{\max }\right)^{2}\right] \mathrm{d} t
\end{aligned}
$$

We define the $n$th moments of the pupil around $u_{\max }$ as

$$
I_{n}^{\prime}=\int_{0}^{1} Q(t) t^{n} \exp \left(i u_{\max } t / 2\right) \mathrm{d} t
$$

To calculate the intensity along the axis we take into account only the terms up to second order in $u^{\prime}=$ $u-u_{\max }$. In this case the axial intensity is approximated as

$$
\begin{aligned}
I\left(0, u^{\prime}\right) \cong & \left|I_{0}{ }^{\prime}\right|^{2}-\operatorname{Im}\left(I_{0}{ }^{\prime *} I_{1}{ }^{\prime}\right) u^{\prime}+(1 / 4) \\
& \times\left[\left|I_{1}\right|^{2}-\operatorname{Re}\left(I_{0}{ }^{\prime} I_{2}{ }^{* *}\right)\right] u^{\prime 2},
\end{aligned}
$$

where $*$ indicates the conjugate operation. For the transverse response we evaluate the point-spread function at the plane corresponding to $u_{\max }$ and expand to second order the transverse response as a function of the transverse coordinate $v$, i.e.,

$$
U\left(v, u_{\max }\right) \cong \int_{0}^{1} Q(t)\left[1-(1 / 4) v^{2} t\right] \exp \left(i u_{\max } t\right) \mathrm{d} t
$$

Then, the transverse intensity can be expressed as

$$
I\left(v, u_{\max }\right) \cong\left|I_{0}\right|^{2}-(1 / 2) \operatorname{Re}\left(I_{0}^{\prime} I_{1}{ }^{\prime *}\right) v^{2} .
$$

We call $u_{0}$ the value of $u^{\prime}$ that corresponds to the center of the parabola defined by Eq. (5), i.e.,

$$
u_{0}=2 \operatorname{Im}\left(I_{0}{ }^{* *} I_{1}{ }^{\prime}\right) /\left[\left|I_{1}\right|^{2}-\operatorname{Re}\left(I_{0}{ }^{\prime} I_{2}{ }^{* *}\right)\right] .
$$

Note that $u_{0}$ is measured from the BIP centered at $u_{\max }$, so its values will be very close to zero for most functions that represent the axial response of an optical system.

Taking into account expressions (5) and (7) and Eq. (8), we generalize the expressions for the axial and transverse superresolution factors, $G_{A}$ and $G_{T}$, respectively, introduced by Sheppard and Hegedus ${ }^{6}$ for amplitude filters and by de Juana et al. ${ }^{8}$ for phase filters. The superresolution factors around $u_{\max }$ result in

$$
\begin{aligned}
& G_{A}=12 \frac{\operatorname{Re}\left(I_{0}{ }^{\prime} I_{2}{ }^{*}\right)-\left|I_{1}\right|^{2}}{\left|I_{0}\right|^{2}-(1 / 2) u_{0} \operatorname{Im}\left(I_{0}{ }^{\prime *} I_{1}{ }^{\prime}\right)}, \\
& G_{T}=2 \operatorname{Re}\left(I_{0}{ }^{\prime} I_{1}{ }^{* *}\right) /\left|I_{0}\right|^{2} .
\end{aligned}
$$

Also we can generalize the Strehl ratio $S$ as

$$
S=\left|I_{0}\right|^{2}-u_{0} \operatorname{Im}\left(I_{0}{ }^{* *} I_{1}{ }^{\prime}\right) .
$$

One can obtain diverse responses by varying the parameters involved in the super-Gaussian function of Eq. (1). As examples, we show how transverse superresolving filters can be obtained in two cases in which the axial gain is very different. We choose a first set of parameters, $a=3, \Omega=0.1$, and $\alpha=2$, and we calculate the axial and the transverse gain as a function of $t_{0}$. The results are shown in Fig. 1(a). The dotted line shows the unitary gain. From this figure it can be observed that the axial gain is symmetrical with (a)

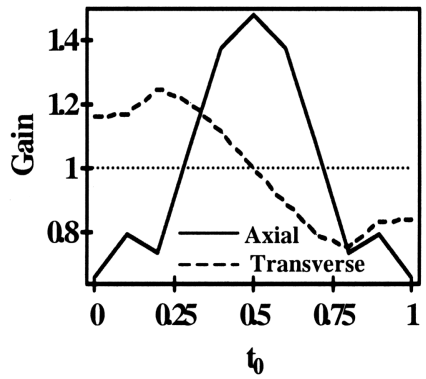

(b)

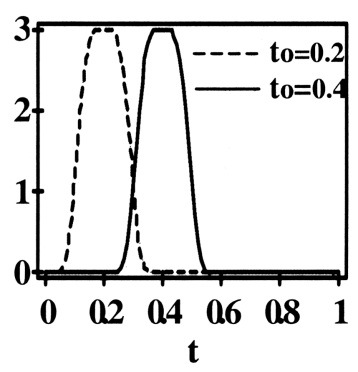

Fig. 1. (a) Axial and transverse gain versus $t_{0}$ for $a=$ $3, \Omega=0.1$, and $\alpha=2$. (b) Phase transmission for filters with distributions centered at $t_{0}=0.2$ and $t_{0}=0.4$. 

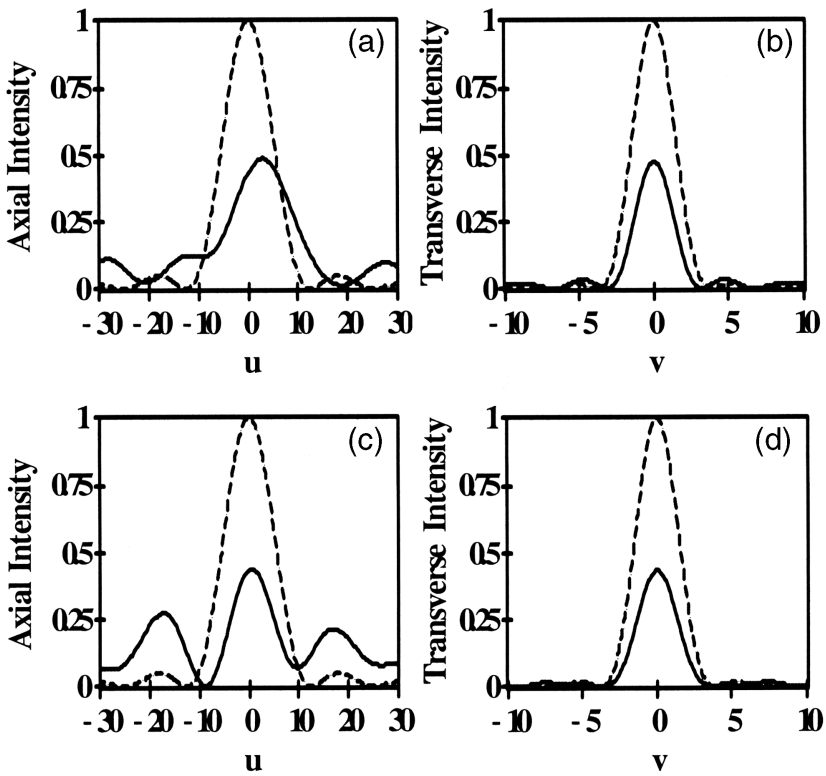

Fig. 2. (a), (c) Axial intensities for $t_{0}=0.2$ and $t_{0}=0.4$ respectively. (b) Transverse intensity for $t_{0}=0.2, u_{\max }=$ $2, S=0.49, G_{T}=1.2$, and $G_{A}=0.7$. (d) Transverse intensity for $t_{0}=0.4, u_{\max }=0.5, S=0.44, G_{T}=1.1$, and $G_{A}=1.4$.

respect to $t_{0}=0.5$ and the transverse gain is antisymmetrical with respect to the same point.

Moreover, we can observe that in the case of $t_{0}<0.5$ the transverse gain is higher than 1 , which implies that the filter acts as a transverse superresolving filter. On the contrary, if $t_{0}>0.5$, the transverse gain is less than 1 and the filter acts as a transverse apodizing filter. In both cases we can choose positions for $t_{0}$ for which the filter acts as either a superresolving or an apodizing axial filter. We have selected two values of $t_{0}$ that illustrate cases in which the transverse response corresponds to a transverse superresolving filter while the axial response is associated with an axial apodizing filter $\left(t_{0}=0.2\right)$ or an axial superresolving filter $\left(t_{0}=0.4\right)$. The phase functions of these filters are represented in Fig. 1(b). The axial and transverse intensities are shown in Fig. 2. Figures 2(a) and 2(c) show the axial intensities for $t_{0}=0.2$ and $t_{0}=0.4$. The maximum values of each distribution result in $u_{\max }\left(t_{0}=0.2\right)=1.99$ and $u_{\max }\left(t_{0}=0.4\right)=0.51$. Fig- ures 2(b) and 2(d) show the transverse intensities, i.e., the point-spread functions. In all cases the dashed curves indicate the response for the pupil without a filter. The Strehl ratio is $S=0.49$ for Fig. 2(b) and $S=0.44$ for Fig. 2(d). We remark that both filters produce a good transverse response. They produce transverse superresolution with high Strehl values and low sidelobes.

In this Letter we have derived the superresolving gains around shifted focus $u_{\max }$ that properly describe the transverse and axial responses of an optical system with a complex transmission function. The examples presented here show that super-Gaussian phase filters with different parameters allow us to obtain a wide range of responses, from superresolving to apodizing filters in either the axial or the transverse direction. The addition of these filters to optical systems can modify their behavior in terms of image formation. We show that the filters can be designed to get the desired feature.

This research was partially financed by Ministerio de Ciencia y Tecnología, Spain, project BFM2000-0036-C02-01. S. Ledesma (e-mail ledesma@df.uba.ar), a member of the Consejo Nacional de Investigaciones Científicas y Técnicas-Argentina, gratefully acknowledges the financial support of the Generalitat de Catalunya, project AIRE 2002-11.

\section{References}

1. T. R. M. Sales and G. M. Morris, J. Opt. Soc. Am. A 14, 1637 (1997).

2. M. D. Levenson, Phys. Today 46(7), 28 (1993).

3. R. Hild, M. J. Yzuel, and J. C. Escalera, Microelectron. Eng. 34, 195 (1997).

4. M. Gu and C. J. R. Sheppard, J. Mod. Opt. 38, 2247 (1991).

5. M. Martínez-Corral, C. Ibáñez-López, and G. Saavedra, Opt. Express 11, 1740 (2003), http:// www.opticsexpress.org.

6. C. J. R. Sheppard and Z. S. Hegedus, J. Opt. Soc. Am. A 5, 643 (1988).

7. J. Ojeda-Castañeda, J. C. Escalera, and M. J. Yzuel, Opt. Commun. 114, 189 (1995).

8. D. M. de Juana, J. E. Oti, V. F. Canales, and M. P. Cagigal, Opt. Lett. 28, 607 (2003).

9. J. Zhai, Y. Yan, D. Huang, M. Wu, and G. Jin, Proc. SPIE 3429, 177 (1998).

10. J. Campos, J. C. Escalera, C. J. R. Sheppard, and M. J. Yzuel, J. Mod. Opt. 47, 57 (2000). 